\title{
TUNNELING CONDUCTIVITY OF SUPERLATTICE GAAS/ALGAAS N-I-P SOLAR CELLS BY MEANS OF THE GENERALIZED KUBO-GREENWOOD FORMULA AND THE GREEN'B FUNCTION
}

\author{
A.C. Varonides, Physics/Electronics Engineering Department
}

University of Scranton, Scranton, PA $18510-4642$
TEL: (717) $941-6290 \quad$ FAX: (717) $941-4085$

\section{ABSTRACT}

We derive, from first principles, an explicit result for the perpendicular conductivity of superlattice solar cells by means of the generalized KuboGreenwood formulas and the Green's function. The advantage of superlattice based solar cells, when compared to their bulk counterparts is that quantization of energy levels takes place within the quantum wells leading to enhancement of conductivity. These energy levels form minibands that allow the carriers to tunnel through the barriers.

\section{Introduction}

An analytic result for the perpendicular tunneling conductivity of multiquantum well solar cells by means of Green's functions is derived. In $p$ $i-n$ structures, the $p$ - and $n$ - regions may be replaced by superlattice contacts to increase $\mathrm{v}_{\text {g }}$. The dark perpendicular conductivity depends on a number of parameters, such as the period of the superlattice, the energy eigensolutions and their widths, and the Fermi level location. Carriers are expected to tunnel through the potential barriers, thus contributing to conductivity along the growth direction. It is possible to derive an explicit result for the tunneling conductivity by generalizing the Kubo-Greenwood formula by means of the causal Green's function. $\sigma_{22}$ (T) is a sum over the discrete spectrum of the superlattice wave number $k_{z}$ and includes the repeat distance $d$ (period), Fermi level $E_{F}$, and miniband widths $\gamma_{n}$.

U.S. Government work not protected by U.S. copyright.

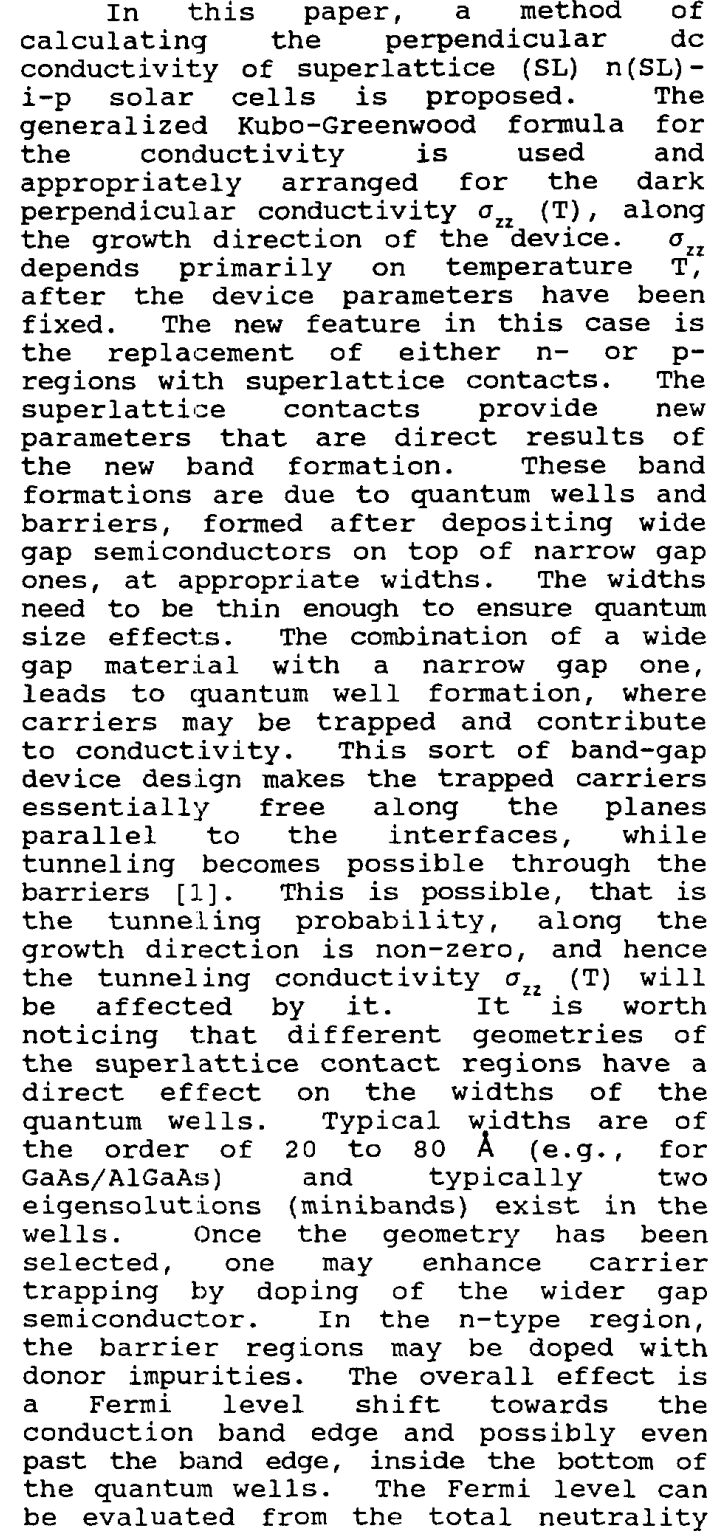


condition. It is already known [2] that Fermi level shifting leads to improvements of $V_{o c}$ (open circuit voltages) and to overall efficiency gains. It is the goal of this paper to show that the dark conductivity can be calculated for $n(S L)-i-p$ superlattices. The materials selected are GaAs (narrow gap) and AlGaAs (wider gap) for the reason that these alloys have been extensively studied and for being crystalline (no defects in the gap).

II. The Rubo-Greenwood Formula of Conductivity

The real part of $\sigma$ is given by [3] the generalized Kubo-Greenwood formula:

$$
\begin{aligned}
& \sigma_{1}(\omega)-\frac{e^{2} \hbar}{\pi m^{2} \Omega} \int d E \frac{f(E)-f(E+\hbar \omega)}{\hbar \omega} \times \\
& \operatorname{Tr}\left\{P_{\alpha} \operatorname{ImG}^{+}(E+\hbar \omega) P_{\alpha} \operatorname{ImG}^{+}(E)\right\}
\end{aligned}
$$

where the subindex is $\alpha=x, y, z, \quad \Omega$ is a normalization volume, $f(E)$ is the Fermi Dirac distribution function, and $G^{+}(E)$ is the causal Green's function:

$$
G^{+}\left(E, k_{z}\right)-\frac{1}{E-\Sigma_{1}+\Sigma_{2}-E\left(k_{z}\right)}
$$

$\Sigma=\Sigma_{1}-\Sigma_{2}$ being the electron self energy, $k$ the superlattice wave number, confined in the superlattice's Brillouin zone.

The perpendicular conductivity $\sigma_{z z}$ ( $\mathrm{z}=$ growth direction) is then:

$$
\sigma_{z z}(\omega, T)-\frac{e^{2} \hbar}{\pi m^{2} \Omega} \int d E \frac{f(E)-f(E+\hbar \omega)}{\hbar \omega} \times
$$

$\operatorname{Tr}\left\{P_{z} \operatorname{ImG}^{+}(E+\hbar \omega) P_{z} \operatorname{ImG}^{+}(E)\right\}$

the DC case $(\omega \rightarrow 0)$ leads to:

$$
\begin{gathered}
\sigma_{z z}(T)-\frac{2 e h^{2}}{\pi \Omega} \int d E\left(-\frac{\partial f(E)}{\partial E}\right) \\
\cdot \sum_{k_{z}}\left|\left\langle k_{z}\left|\frac{P_{z}}{m}\right| k_{z}\right\rangle\right|^{2}\left|\operatorname{ImG}^{+}\left(E_{1} k_{z}\right)\right|^{2}
\end{gathered}
$$

The sum is over the discrete spectrum of $k_{2}$ (with a superlattice period d), which is confined in the superlattice's Brillouin zone:

$$
-\frac{\pi}{d} \leq \mid k_{z} \leq \frac{\pi}{d}
$$

and is given by the Born-von Karman boundary combination [4]

$$
K_{z}^{(j)}=-\frac{\pi}{d}+\frac{2 \pi j}{N \cdot d}, j=0,2, \ldots, N
$$

where $\mathrm{N}$ is the number of periods of the superlattice.

Referring to (4), we can show that

$$
\left\langle\mathrm{K}_{\mathrm{z}}\left|\frac{\mathrm{P}_{\mathrm{z}}}{\mathrm{m}}\right| \mathrm{k}_{\mathrm{z}}\right\rangle-\frac{1}{\hbar} \frac{\partial \mathrm{E}\left(\mathrm{k}_{\mathrm{z}}\right)}{\partial \mathrm{k}_{\mathrm{z}}}=\mathrm{v}_{\mathrm{z}}\left(\mathrm{k}_{\mathrm{z}}\right)
$$

where $v_{2}\left(k_{z}\right)$ is the group velocity. For moderate dopings

$$
f(E) \approx \exp \left(-\frac{E-E_{F}}{k T}\right)
$$

Equation (4) becomes

$$
\sigma_{z z}(T)-\frac{2 e^{2} \hbar}{\pi \Omega} \int d E\left(-\frac{\partial f}{\partial E}\right) \sum_{k_{2}}\left(\frac{1}{\hbar} \frac{\partial E}{\partial k_{z}}\right)^{2} x
$$

$\left|\operatorname{ImG}^{+}\right|^{2}$

where

$$
\left|\operatorname{ImG}^{+}\right|^{2}-\frac{\Sigma_{2}{ }^{2}}{\left[\left(E-\Sigma_{1}-E\left(k_{2}\right)\right)^{2}+\Sigma_{2}^{2}\right]^{2}}
$$
Inserting (9) in (8) and evaluating $-\frac{\partial f}{\partial E}$
through (7), at the limit $\omega \rightarrow 0$ one obtains:

$\sigma_{z z}(T)-\frac{2 e^{2} \hbar}{\pi \Omega} \int d E\left[(k T)^{-1} e^{\frac{-\left(E-E_{z}\right)}{k T}}\right] x$

$$
\sum_{\mathrm{k}_{\mathrm{z}}} \frac{1}{\hbar^{2}}\left(\frac{\partial \mathrm{E}}{\partial \mathrm{k}_{\mathrm{z}}}\right)^{2} \frac{\boldsymbol{\Sigma}_{2}{ }^{2}}{\left\{\left(\mathrm{E}-\boldsymbol{\Sigma}_{1}-\mathrm{E}\left(\mathrm{k}_{\mathrm{z}}\right)\right)^{2}+\boldsymbol{\Sigma}_{2}{ }^{2}\right\}^{2}}
$$

where

$$
E\left(k_{z}\right)-E_{o}+\gamma_{n} \cos k_{z} \alpha
$$


(11) is the superlattice dispersion relation, and $\gamma_{n}$ is the width of the nth miniband.

Since

$$
\left(\frac{\partial E}{\partial k_{z}}\right)^{2}-d^{2}\left(\gamma_{n}{ }^{2}-\left(E\left(k_{z}\right)-E_{0}\right)^{2}\right)
$$

(10) becomes

$$
\begin{aligned}
& \sigma_{z z}(T)-\left(\frac{2 e^{2} d^{2} \Sigma_{2}^{2}}{(k T) \hbar \pi \Omega}\right) \sum_{k_{z}}\left[\gamma_{n}{ }^{2}-\left(E\left(k_{z}\right)-E_{o}\right)^{2}\right] x \\
& \int \frac{d E \exp \left(-\frac{E-E_{F}}{k T}\right)}{\left\{\left[\left(E-E_{F}\right)+\left(E_{F}-\Sigma_{1}\right)-E\left(k_{z}\right)\right]^{2}+\Sigma_{2}{ }^{2}\right\}^{2}}
\end{aligned}
$$

Neglecting $\Sigma_{2}{ }^{2}$, in the denominator, and letting $E_{F}-\Sigma_{1} \sim E_{F}$ (weak scattering limit) [3] the function to be integrated becomes

$$
\frac{\exp \left(-\frac{E-E_{F}}{k T}\right)}{\left[\left(E-E_{F}\right)+E_{F}-E\left(k_{z}\right)\right]^{4}}
$$

Carrying the integral in (13) through (14) leads to the following explicit: result for $\sigma_{z z}(T)$ :

$$
\begin{aligned}
\sigma_{z z}(T)= & \left(\frac{2 e^{2} d^{2} \Sigma_{2}^{2}}{(k T) \hbar \pi \Omega}\right) x \\
& \sum_{k_{z}}\left[\gamma_{n}{ }^{2}-\gamma_{n}{ }^{2} \cos ^{2} k_{z} d\right] x \\
& \exp \left(-\frac{\left(E_{o}-E_{F}\right)}{k T}\right) \Phi\left(\gamma_{n}, k_{z}, d, k T\right)
\end{aligned}
$$

where

$$
\begin{aligned}
\Phi- & {\left[-\left(6(k T)^{2} \gamma_{n} \cos k_{z} d\right)^{-1}\right.} \\
& -\left(6 k T \gamma_{n}^{2} \cos ^{2} k_{z} d\right)^{-1} \\
& -\left(3 \gamma_{n}^{2} \cos ^{3} k_{z} d\right)^{-1} \\
& +\frac{1}{6(k T)^{3}} \exp \left(-\frac{\gamma_{n} \cos k_{z} d}{k T}\right)
\end{aligned}
$$

$$
\left.E \dot{E}\left(-\frac{y_{n} \cos k_{z} d}{k T}\right)\right]
$$

where $E i$ is the exponential integral of

$$
\left(-\frac{\gamma_{n} \cos k_{z} d}{k T}\right)
$$

Notice that for (15) not to be zero, cos $k_{2} d$ must not be \pm 1 , which leads to the fact that $j$ must not be either 0 or $N$ (see (5)).

$$
\text { The index } n \text { refers to the } n t h
$$
miniband in the wells.

From the treatment above, it is found that the dark conductivity of a superlattice solar cell of the type $n(S L)-i-p$ can be calculated in terms of the Fermi level position $E_{f}$, eigenenergies inside the quantum wells, their widths $\gamma_{n}$, the period d of the superlattice. The Fermi level in (15) is found with very good accuracy by means of the neutrality condition for composite superlattices $[5,6]$ as shown in Fig. 1. As it is usually the case, the superlattice parameters are at the designer's disposal and the general formula (15) may lead to values of $\sigma_{2 z}(T)$. The A.C. case $(\omega \neq 0)$ (see (1)) is under study. GaAs/AlgaAs thin heterojunctions are good candidates, since these junctions have been studied extensively.

\section{REFERENCES}

(1) A.C. Varonides. "n (GaAs/AlGaAs)-i (GaAs) - $p$ (GaAs) Novel Multiquantum Well Solar cell Structures," presented at the 11th European Photovoltaic Conference, Montreux, Switzerland, 12-16 October 1992, Proceedings pp 909-912.

(2) A.C. Varonides and A. Rothwarf. "a-Si:h (Alloy $p-i-n$ Superlattice Solar cell contacts," IEEE Transactions on Electron Devices, vol. 39, No. 10, pp 2284-2289, 1992 . 
(3) E.N. Economou. "Green's Functions in Quantum Physics," 2nd ed., Springer-Verlag, 1983.

(4) G. Bastard. "Wave mechanics applied to semiconductor heterostructures," Halsted Press, 1988.

(5) A.C. Varonides and A. Rothwarf. "A New High open Circuit Voltage $P(a-$ SiN:H)-I(a-Si:H)-N(a-SiC:H/a-Si:H) superlattice solar cell," Transactions of the loth European Photovoltaic Solar Energy Conference \& Exhibition, Lisbon, Portugal, 8-12 April 1991, pp 416419.

(6) A. Rothwarf and A.C. Varonides. "Superlattice Contact Layers for High open Circuit Voltage a-Si:H Solar Cells," 21st IEEE Photovoltaic specialists Conference, Vol. II, pp 1555-1559, 1990

n+-AlGaAs wide gap semiconduct or

GaAs narrow gap semiconductor

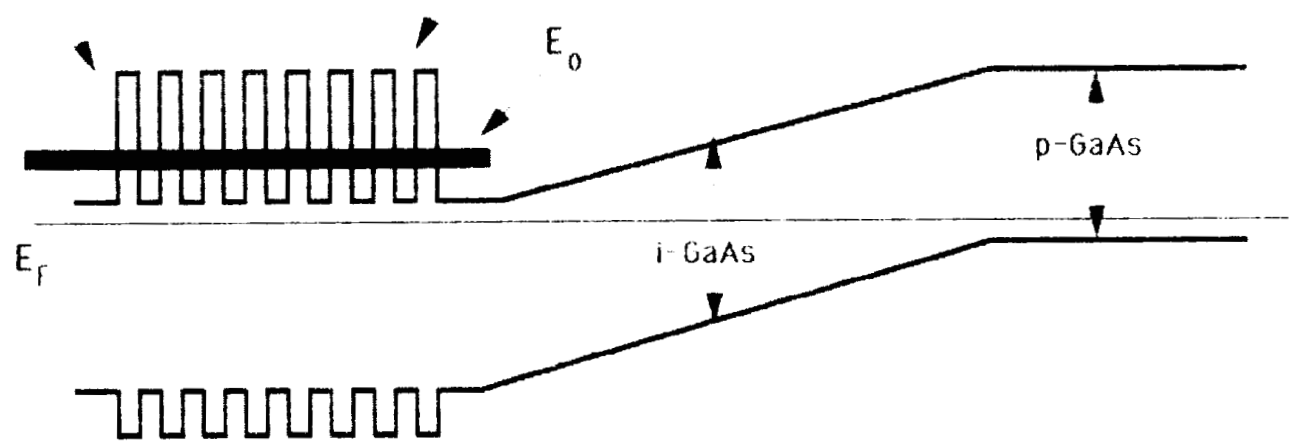

tig. - Proposed superlattice solar cell structure, typically AlGaAs/GaAs, for which the dark perpendicular conductivity is evaluated $\mid 1\}$. 